\title{
Spectral Properties of Abdominal Tissues on Dual-energy Computed Tomography and the Effects of Contrast Agent
}

\author{
DIANA A. KREUL ${ }^{1,2}$, RAHEL A. KUBIK-HUCH ${ }^{1 *}$, JOHN FROEHLICH ${ }^{3}$, \\ MICHAEL J. THALI ${ }^{2}$ and TILO NIEMANN ${ }^{1 *}$ \\ ${ }^{1}$ Institute of Radiology, Kantonsspital Baden, Baden, Switzerland; \\ ${ }^{2}$ Institute of Forensic Medicine, Zürich, Switzerland; \\ ${ }^{3}$ Guerbet $A G$, Zürich, Switzerland
}

\begin{abstract}
Background/Aim: Multiparametric dual energy comptuted tomography (CT) imaging allows for multidimensional tissue characterization beyond the measurement of Hounsfield units. The purpose of this study was to evaluate multiple imaging parameters for different abdominal organs in dual energy CT (DECT) and analyze the effects of the contrast agent on these different parameters and provide normal values for characterization of parenchymatous organs. Patients and Methods: This retrospective analysis included a total of 484 standardized DECT scans of the abdomen. Hounsfield Units $(H U)$, rho (electron density relative to water), $Z_{\text {eff }}$ (effective atomic number) and FF (fat fraction) were evaluated for liver, spleen, kidney, muscle, fat-tissue. Independent generalized estimation equation models were fitted. Results: In DECT imaging there is only little difference in mean $H U_{\text {mixed }}$ for parenchymatous

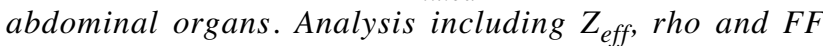
allows for better discrimination while a large overlap remains for liver, spleen and muscle. Including multidimensional analysis and the effects of contrast medium further enhances tissue characterization. Small differences remain for liver and spleen. Conclusion: Organ characterization using multiparametric dual energy CT analysis is possible. An increased number of parameters obtained from DECT improves organ
\end{abstract}

This article is freely accessible online.

*These Authors contributed equally to this study.

Correspondence to: Tilo Niemann, Department of Radiology, Kantonsspital Baden in Baden, Im Ergel 1, 5404 Baden, Switzerland. Tel: +41 564862111, Fax: +41 564863829, e-mail: tilo.niemann@ksb.ch

Key Words: Computed X-ray tomography, dual-energy computed tomography, abdominal imaging. characterization. To our knowledge this is the first attempt to provide normal values for characterization of parenchymatous organs.

The differentiation of tissue and organs on single-energy computed-tomography (CT) is performed according to differences in X-ray attenuation, which in the case of CT is measured as Hounsfield units (HU) relative to the attenuation of pure water $(1,2)$. The CT number is influenced by the effective atomic number $\left(\mathrm{Z}_{\text {eff }}\right)$ and the electron density of each material $\left(\varrho_{e}\right)$. As the effective atomic number is dependent to spectral properties, a change in X-ray energy leads to a change in the resulting Hounsfield units for a tissue accordingly (3-6).

The basic idea of dual-energy CT (DECT) is to apply two different X-ray energies and hence take advantage of the different spectral properties. This method has become established over recent years and offers the possibility of new and better tissue characterizations (7-15). By acquiring two CT datasets either simultaneously or one immediately after another, DECT enables the concurrent acquisition of two attenuation maps at low- and high-energy spectrums (10, 15-17). Although different tissues may show quite similar attenuation (in terms of CT numbers) at a certain energy level $(7,8)$, they may show large differences in attenuation at other energy levels because of their individual electron binding energies $(9,10,12)$. The two main reasons for this effect are Compton-scattering and photoelectric-absorption $(5,6,11-13,15,18)$. Compton scatter is nearly independent of the photon-energy, depending primarily on the electron density of the material. It occurs predominately at high energies. However, photoelectric-absorption is highly dependent on the electron binding energy, which in turn is highly dependent on the atomic number of the examined element $(3-6,13,18)$.

The probability of photoelectric-absorption increases substantially when the energy of a photon approaches the individual binding energy of a material. On the basis of these 
effects, different tissues may show large differences in their probabilities for photoelectric interactions, and the individual spectral characteristics may assist in the characterization of different tissues on DECT (9-12, 14-18).

The purpose of this study was to evaluate multiparametric analysis of different tissues on DECT-scans implemented in a standardized clinical routine setting over the course of a year. The tissues evaluated were abdominal organs (liver, spleen, kidney) and muscle, as all these materials have quite similar elemental compositions and may be challenging for automated segmentation methods using single-energy CT images. Additionally, abdominal fat tissue was measured, which should be easier to separate from soft tissue because of its lower density (1). By using dedicated software (syngo.via ${ }^{\circledR}$ Dual Energy, Siemens Healthineers, Forchheim, Germany), CT images acquired at two different energy levels can be decomposed into two new material-specific images by the application of spectral processing techniques. The results can be presented as either $Z_{\text {eff }}$ and $\varrho_{e}$ images, or as one image representing high atomic number material (e.g., iodine) and the other image representing soft tissue, which also permits analysis of the fat fraction. A future idea is to create automated segmentation techniques for abdominal imaging, using dedicated trained software for the automatic recognition of abdominal organs.

We, furthermore, analyzed the effect of a contrast agent on the different DECT parameters of each organ, as this information could contribute to future studies researching pathological changes in organs. It is likely that angiopathicischemic lesions with reduced blood circulation or blood clots in the vascular system of an organ will lead to low contrast agent uptake in the respective organ.

\section{Patients and Methods}

This retrospective study was performed using routinely acquired clinical DECT-scans of human participants. The requirement for informed patient consent for this retrospective study was waived by the local ethics committee (EKNZ 2018-01641). In addition, written informed consent was obtained for the research use of the clinical data of each participant.

Study population. This study was performed at a public hospital using data acquired from patients between February 2018 and February 2019. The institution has subspecialty-trained radiologists dedicated to the analysis of CT and DECT images. The inclusion criteria for subject selection were patients above 18 years who provided written informed consent and underwent one of two defined standardized DECT-protocols. All patients were referred for DECT due to suspected kidney/ureter stones or work-up of hematuria. Sixteen DECT scans from 16 patients were excluded because the patients received two non-contrast and two contrast-enhanced DECT scans during the study period. In total, imaging data from 331 different patients were included in the study.
Imaging modalities. Dual-energy CT was performed with a SOMATOM Definition Flash second-generation dual-source CT scanner (Siemens Healthineers). Two standardized DECT protocols for the abdomen (non-contrast and contrast-enhanced) were included in the study. The standard-of-care imaging was a noncontrast DECT scan of the abdomen in patients with suspected kidney/ureter stones and a combined non-contrast and venous-phase contrast DECT scan of the abdomen for work-up of hematuria. Axial spiral datasets of the abdomen were acquired during a single breath-hold.

The scanning and reconstruction parameters for the unenhanced images were: $\mathrm{kV} \mathrm{100/Sn} \mathrm{140,} \mathrm{reference} \mathrm{mAs} \mathrm{70,} \mathrm{rotation} \mathrm{time} 0.5 \mathrm{~s}$, pitch 0.7 , collimation $32 \times 0.6 \mathrm{~mm}$, and reconstruction $1.5 \mathrm{~mm}$. The scanning and reconstruction parameters for the contrast-enhanced images were: $\mathrm{kV} \mathrm{100/Sn} \mathrm{140,} \mathrm{reference} \mathrm{mAs} \mathrm{94,} \mathrm{rotation} \mathrm{time} 0.5 \mathrm{~s}$, pitch 1.2 , collimation $32 \times 0.6 \mathrm{~mm}$, and reconstruction $1 \mathrm{~mm}$.

Image evaluation. All dual-energy images were evaluated using syngo.via ${ }^{\circledR}$ software (syngo Dual Energy, Siemens Healthcare GmbH 2009-2018, Version 05.01.0000.0030, Erlangen, Germany). Standardized segments of each organ were measured by one reader using standardized regions of interest (ROIs). The measured DECTparameters included $\mathrm{HU}$ values of the mixed $100 \mathrm{kV}$ and $140 \mathrm{Sn}$ $\mathrm{kV}$ images $\left(\mathrm{HU}_{\text {mixed }}\right.$; representing a $120 \mathrm{kV}$ image), electron density relative to water $\left(\varrho_{e}\right)$, effective atomic number $\left(Z_{e f f}\right)$, and fat fraction (FF). Image analysis and measurement processing were performed under the supervision of a board-certified radiologist.

The readers were free to choose optimal window settings for the image analysis. After visual assessment, the parameters were measured using ROIs set in the liver, spleen, kidney, muscle, and fat tissue. All parameters were evaluated on DECT-scans with and without contrast agent. For each tissue, at least two ROIs of between 0.5 and $4 \mathrm{~cm}^{2}$ were placed on axial image views. Contact with macroscopic vessels or organ capsules were avoided. In the liver, three ROIs were placed in the periphery of the right hepatic lobe (hepatic segments VIII, VI, V) and one ROI was placed in the left hepatic lobe (III). In the spleen, one ROI was set in the upper half and one in the lower half. In each kidney, three ROIs were set in the cortex between the upper and lower pole, avoiding contact with the renal pelvis. For muscle measurements, the psoas, obturatorius, sartorius, or gluteus muscles were measured on both sides, avoiding muscles showing signs of fatty degeneration or atrophy in the image reporting. Fat tissue was measured in subcutaneous adipose tissue, preferably in the lumbar region. As several ROIs were placed in each organ, the measurements were averaged for each participant and organ.

Figure 1 shows the application profiles used in syngo.via ${ }^{\circledR}$ software. Measurements of attenuation were taken on default view images. Afterwards, the specific DECT-parameters $\left(\varrho_{e}, Z_{\text {eff }}, F F\right)$ of each tissue were extracted using the application profiles "Rho/Z" and "fat map" (subcategories of the application profile "Liver VNC"). Both profiles create material decomposition images and display all structures in a color-coded overlay. The application class "Liver VNC" is based on an iodine subtraction using a material decomposition algorithm encoding the iodine of the contrast agent $(19,20)$. Consequently, every voxel is decomposed into iodine, fat, and soft tissue maps. The Liver VNC application offers the possibility of quantifying the fat fraction of each material in a certain ROI using the sub-algorithm "fat map". The application class "Rho/Z" provides $\mathrm{Z}_{\text {eff }}$ directly, where Rho is encoded in HU-like units $\left(\mathrm{HU}_{\mathrm{Rho}}\right)$, and is 

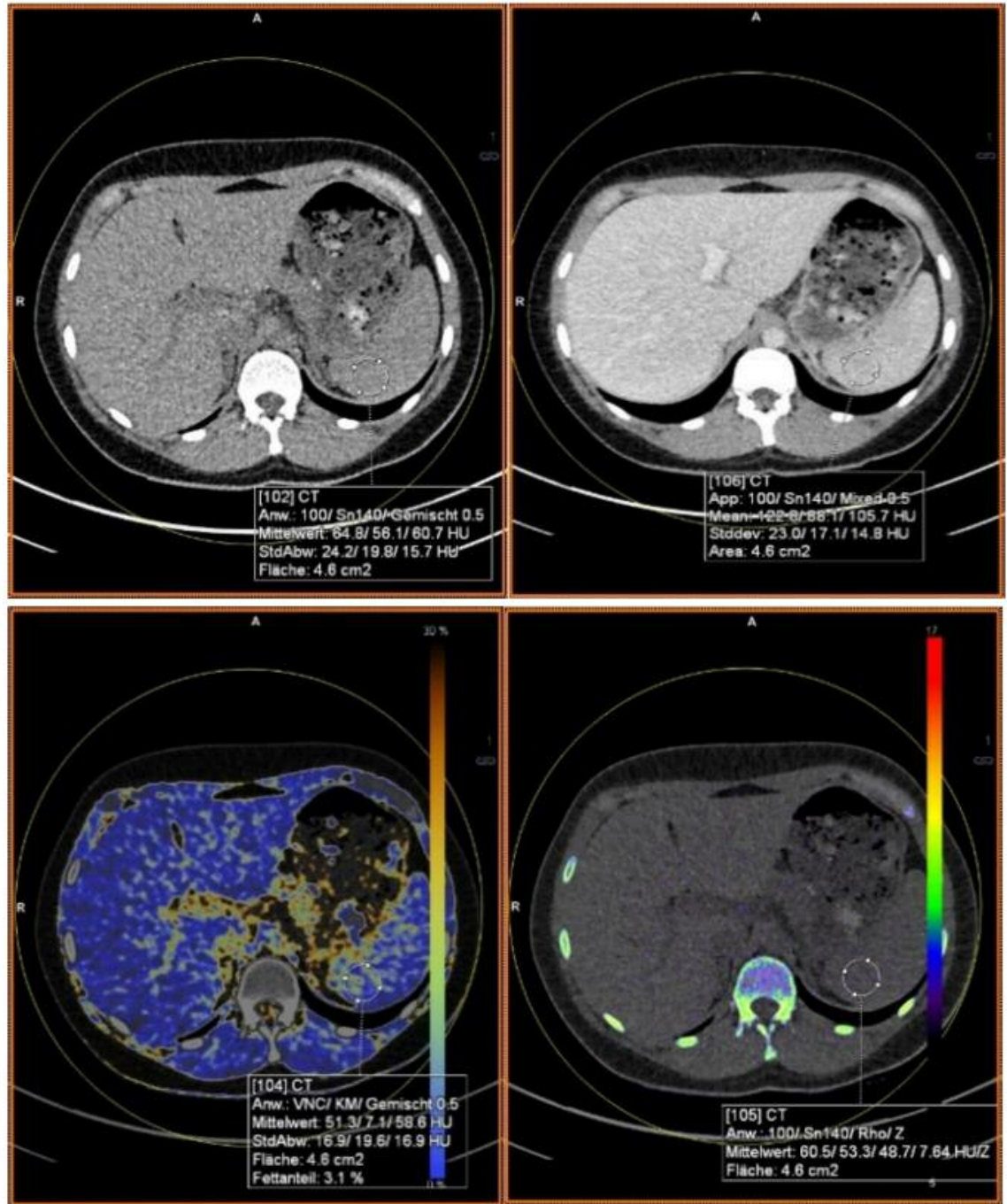

Figure 1. Three different application profiles in syngo.via ${ }^{\circledR}$ Dual Energy (Siemens Healthineers, Erlangen, Germany) for multiparametric analysis. Top left: Unenhanced DECT scan for measurements of CT number. HU mixed, Hounsfield units derived from mixed images of $100 \mathrm{kV}$ and $140 \mathrm{Sn} \mathrm{kV}$, representing a $120 \mathrm{kV}$ image. Top right: contrast-enhanced DECT scan. Bottom left: Special application profile "Liver VNC" creating virtual noncontrast images for quantification of fat fraction [FF (\%)]. Bottom right: Profile "Rho/Z" applied to an unenhanced DECT scan for measurement of

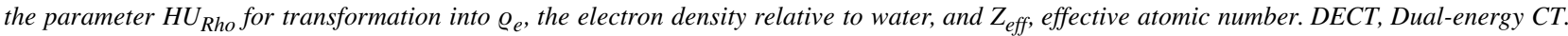

converted into electron density values by applying the linear relationship from Saito et al., as shown below (21):

$$
\rho_{e}=\frac{H U}{1000}+1
$$

Statistical analysis. Statistical analysis was performed using R software version 4.0.0 (2020-04-24). Summary statistics for each organ were created separately, with and without contrast agent, and included the number of observations on which the calculations were based, minimum, $1^{\text {st }}$ and $3^{\text {rd }}$ quartile, median, mean, maximum, standard deviation, and interquartile range. Potential interactions between organs and contrast agent were taken into account by performing analyses separately with and without contrast agent. Independent generalized estimation equation models (GEEs) were fitted to quantify the differences between the organs with respect to the parameters $\mathrm{HU}_{\text {mixed }}$, $F F, \varrho_{e}$, and $Z_{\text {eff }}$, and to assess the effects of contrast agent on the parameters. The reported (unadjusted) 95\% Wald-type confidence intervals are based on the sandwich variance estimator, thus taking intraindividual correlation into account. As there were at most two measurements, the choice of correlation structure was irrelevant.

\section{Results}

Study participants. The full data set consisted of a total of 2,420 measurements from 331 patients. One hundred and seventy-eight patients underwent DECT with contrast agent 


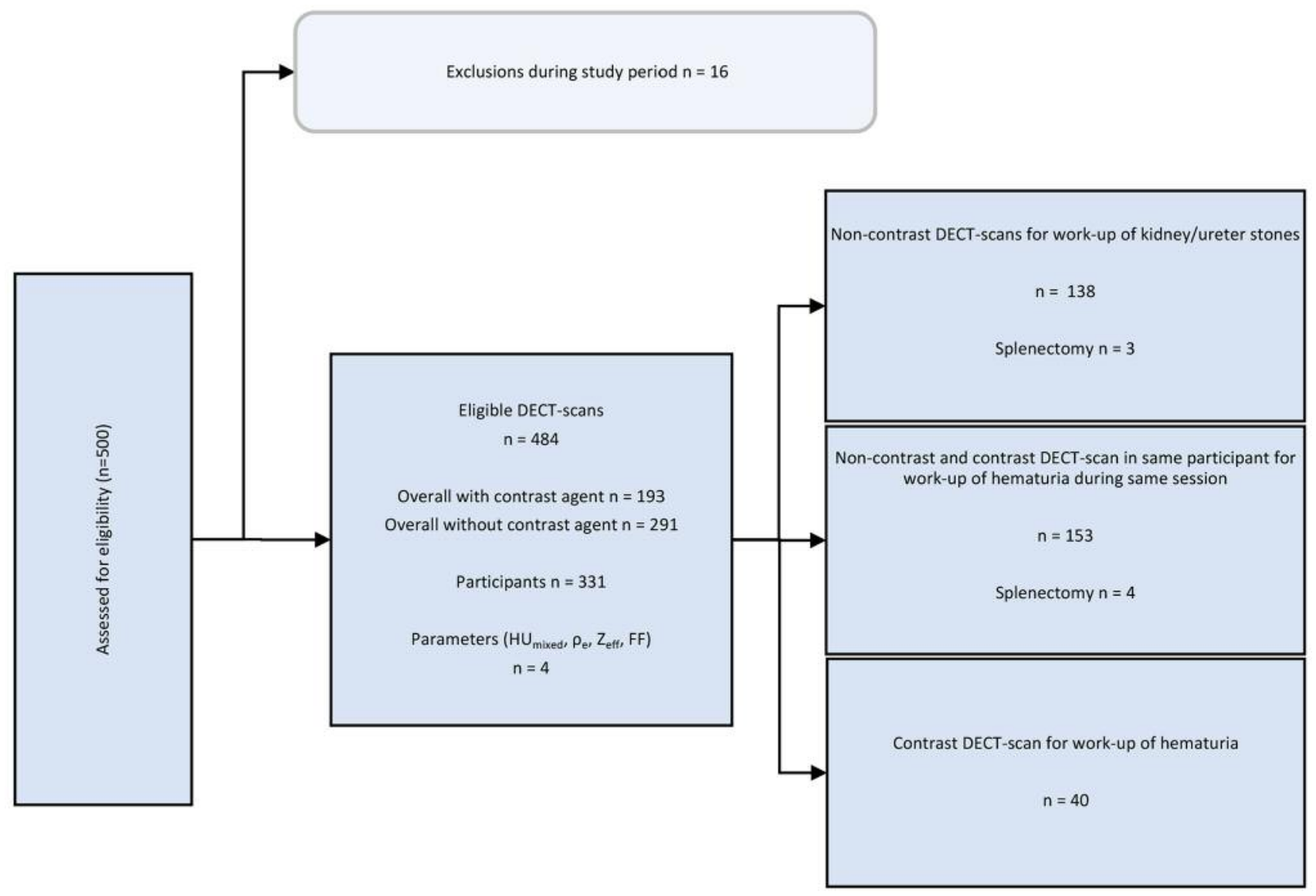

Total no. of measurements $n=2,420$

Figure 2. Flowchart showing the number of participants undergoing the different DECT scan protocols. DECT, Dual-energy CT.

$(n=40)$ or without contrast agent $(n=138)$, whereas 153 patients underwent DECT scans with and without contrast agent during the same session. In total, we report on 291 non-contrast DECT scans and 193 contrast-enhanced DECT scans. Measurements of the spleen were not possible in some cases because of splenectomy. Figure 2 presents a flow chart of the patient selection process.

Data analysis. All parameters were successfully measured in all regions of interest in each organ or tissue. The measured $\mathrm{HU}_{\mathrm{Rho}}$ values of the "Rho/Z" application profile were successfully transformed into the electron density relative to water $\left(\varrho_{e}\right)$ using the formula described by Saito et al. (21). The color-coded fat overlay images of the application profile "Liver VNC - fat map" showed visually clear differences between the different included tissues, with different levels of fat content. Abdominal organs were displayed in blue (no or low fat) and fat tissue in yellow to black (fat).

Statistical analysis. The summary statistics for the DECT scans without contrast agent, representing the number of observations on which the calculations are based, minimum, $1^{\text {st }}$ and $3^{\text {rd }}$ quartiles, median, mean, maximum, standard deviation, and interquartile range, are shown in Table I. Summary statistics for DECT scans with contrast agent are shown in Table II.

The effect of contrast agent on the variables was separately assessed for each organ, and the results are shown in Table III. We report the estimated difference between noncontrast and contrast-enhanced DECT scans for each 
Table I. Summary statistics of dual energy CT (DECT) scans without contrast agent.

\begin{tabular}{|c|c|c|c|c|c|c|c|c|c|c|}
\hline Organ & Parameter & n.valid & $\min$ & Q1 & median & mean & Q3 & $\max$ & SD & IQR \\
\hline \multirow[t]{4}{*}{ Spleen } & FF & 288 & -19.50 & -3.88 & -1.50 & -1.83 & 0.25 & 15.20 & 3.96 & 4.06 \\
\hline & $\mathrm{HU}_{\text {mixed }}$ & 288 & 38.30 & 52.00 & 54.95 & 54.50 & 56.98 & 63.95 & 4.03 & 4.96 \\
\hline & @e & 288 & 1.04 & 1.05 & 1.05 & 1.05 & 1.06 & 1.06 & 0.00 & 0.00 \\
\hline & $Z_{\text {eff }}$ & 288 & 6.69 & 7.28 & 7.38 & 7.36 & 7.43 & 8.07 & 0.14 & 0.15 \\
\hline \multirow[t]{4}{*}{ Liver } & FF & 291 & -17.85 & -9.00 & -5.60 & -4.60 & -1.25 & 18.10 & 6.56 & 7.72 \\
\hline & $\mathrm{HU}_{\text {mixed }}$ & 291 & 27.60 & 54.68 & 60.70 & 58.97 & 65.18 & 73.98 & 8.49 & 10.39 \\
\hline & $\varrho_{e}$ & 291 & 1.03 & 1.05 & 1.06 & 1.06 & 1.07 & 1.07 & 0.01 & 0.01 \\
\hline & $Z_{\text {eff }}$ & 291 & 6.60 & 7.25 & 7.33 & 7.32 & 7.39 & 7.72 & 0.13 & 0.13 \\
\hline \multirow[t]{4}{*}{ Kidneys } & $\mathrm{FF}$ & 291 & -9.08 & 7.20 & 10.62 & 10.64 & 13.77 & 29.53 & 5.38 & 6.53 \\
\hline & $\mathrm{HU}_{\text {mixed }}$ & 291 & 21.60 & 29.58 & 32.25 & 32.33 & 35.07 & 44.45 & 3.92 & 5.47 \\
\hline & $\varrho_{e}$ & 291 & 1.02 & 1.03 & 1.03 & 1.03 & 1.04 & 1.04 & 0.00 & 0.01 \\
\hline & $Z_{\text {eff }}$ & 291 & 6.95 & 7.35 & 7.43 & 7.45 & 7.55 & 8.11 & 0.17 & 0.20 \\
\hline \multirow[t]{4}{*}{ Fat tissue } & FF & 291 & 66.15 & 93.45 & 96.35 & 95.57 & 99.05 & 105.95 & 5.44 & 5.55 \\
\hline & $\mathrm{HU}_{\text {mixed }}$ & 291 & -83.30 & -74.30 & -71.85 & -70.35 & -68.80 & -32.60 & 6.73 & 5.45 \\
\hline & $\varrho_{e}$ & 291 & 0.92 & 0.93 & 0.93 & 0.93 & 0.93 & 0.97 & 0.01 & 0.01 \\
\hline & $Z_{\text {eff }}$ & 291 & 4.61 & 5.30 & 5.55 & 5.53 & 5.76 & 6.80 & 0.34 & 0.46 \\
\hline \multirow[t]{4}{*}{ Muscle } & FF & 291 & -20.50 & -10.20 & -6.90 & -6.79 & -3.40 & 13.15 & 5.43 & 6.75 \\
\hline & $\mathrm{HU}_{\text {mixed }}$ & 291 & 34.15 & 55.00 & 58.75 & 57.90 & 62.00 & 70.25 & 6.50 & 6.93 \\
\hline & $\varrho_{e}$ & 291 & 1.03 & 1.05 & 1.06 & 1.06 & 1.06 & 1.07 & 0.01 & 0.01 \\
\hline & $\mathrm{Z}_{\mathrm{eff}}$ & 291 & 6.18 & 7.17 & 7.30 & 7.28 & 7.40 & 9.09 & 0.22 & 0.23 \\
\hline
\end{tabular}

n.valid represents the number of observations on which the calculations were based. Min, Minimum; Q1, $1^{\text {st }}$ quartile; Q3, $3^{\text {rd }}$ quartile; max, maximum; SD, standard deviation; IQR, interquartile range; $\mathrm{HU}_{\text {mixed }}$, Hounsfield units derived from mixed tube voltages of 100 and $140 \mathrm{kVp}$ representing $120 \mathrm{kV} ; \varrho_{\mathrm{e}}$, electron density relative to water; $Z_{\mathrm{eff}}$, effective atomic number; FF, fat fraction.

Table II. Summary statistics of dual energy CT (DECT) scans with contrast agent.

\begin{tabular}{|c|c|c|c|c|c|c|c|c|c|c|}
\hline Organ & Parameter & n.valid & $\min$ & Q1 & median & mean & Q3 & $\max$ & SD & IQR \\
\hline \multirow[t]{4}{*}{ Spleen } & $\mathrm{FF}$ & 189 & -16.60 & -9.75 & -5.55 & -6.03 & -2.65 & 9.20 & 4.77 & 7.10 \\
\hline & $\mathrm{HU}_{\text {mixed }}$ & 189 & 48.80 & 58.00 & 60.65 & 61.62 & 64.50 & 74.30 & 4.95 & 6.50 \\
\hline & $Q_{e}$ & 189 & 1.05 & 1.06 & 1.06 & 1.06 & 1.06 & 1.07 & 0.00 & 0.01 \\
\hline & $Z_{\text {eff }}$ & 189 & 7.75 & 8.27 & 8.40 & 8.42 & 8.60 & 9.14 & 0.25 & 0.33 \\
\hline \multirow[t]{4}{*}{ Liver } & FF & 193 & -19.75 & -11.65 & -7.38 & -6.55 & -3.15 & 17.78 & 7.23 & 8.50 \\
\hline & $\mathrm{HU}_{\text {mixed }}$ & 193 & 31.50 & 57.75 & 64.95 & 62.76 & 69.20 & 79.40 & 9.24 & 11.45 \\
\hline & $Q_{e}$ & 193 & 1.03 & 1.06 & 1.06 & 1.06 & 1.07 & 1.08 & 0.01 & 0.01 \\
\hline & $\mathrm{Z}_{\text {eff }}$ & 193 & 7.56 & 8.25 & 8.36 & 8.40 & 8.52 & 9.38 & 0.27 & 0.27 \\
\hline \multirow[t]{4}{*}{ Kidneys } & FF & 193 & -1.40 & 7.33 & 10.00 & 10.30 & 13.07 & 26.27 & 4.88 & 5.74 \\
\hline & $\mathrm{HU}_{\text {mixed }}$ & 193 & 25.47 & 35.82 & 38.35 & 38.97 & 41.90 & 52.08 & 4.86 & 6.08 \\
\hline & $Q_{\mathrm{e}}$ & 193 & 1.03 & 1.04 & 1.04 & 1.04 & 1.04 & 1.05 & 0.00 & 0.01 \\
\hline & $\mathrm{Z}_{\mathrm{eff}}$ & 193 & 8.47 & 9.78 & 9.98 & 9.96 & 10.18 & 10.71 & 0.38 & 0.40 \\
\hline \multirow[t]{4}{*}{ Fat tissue } & FF & 193 & 64.20 & 95.15 & 97.95 & 96.85 & 100.15 & 104.8 & 5.86 & 5.00 \\
\hline & $\mathrm{HU}_{\text {mixed }}$ & 193 & -83.05 & -75.65 & -73.05 & -71.47 & -69.55 & -32.75 & 7.01 & 6.10 \\
\hline & $Q_{e}$ & 193 & 0.92 & 0.92 & 0.93 & 0.93 & 0.93 & 0.97 & 0.01 & 0.01 \\
\hline & $\mathrm{Z}_{\mathrm{eff}}$ & 193 & 4.85 & 5.69 & 5.96 & 5.98 & 6.18 & 7.77 & 0.44 & 0.48 \\
\hline \multirow[t]{4}{*}{ Muscle } & FF & 193 & -14.15 & -8.25 & -5.60 & -4.84 & -2.15 & 7.85 & 4.65 & 6.10 \\
\hline & $\mathrm{HU}_{\text {mixed }}$ & 193 & 38.90 & 52.95 & 57.00 & 57.04 & 61.80 & 69.55 & 6.08 & 8.85 \\
\hline & $\varrho_{e}$ & 193 & 1.04 & 1.05 & 1.06 & 1.06 & 1.06 & 1.07 & 0.01 & 0.01 \\
\hline & $\mathrm{Z}_{\text {eff }}$ & 193 & 6.35 & 7.32 & 7.55 & 7.48 & 7.72 & 8.04 & 0.31 & 0.39 \\
\hline
\end{tabular}

n.valid represents the number of observations on which the calculations were based. Min, Minimum; Q1, $1_{\mathrm{st}}$ quartile; Q3, $3_{\text {rd }}$ quartile; max, maximum; SD, standard deviation; IQR, interquartile range; $\mathrm{HU}_{\text {mixed }}$, Hounsfield units derived from mixed tube voltages of 100 and $140 \mathrm{kVp}$ representing $120 \mathrm{kV} ; \varrho_{\mathrm{e}}$, electron density relative to water; $\mathrm{Z}_{\mathrm{eff}}$, effective atomic number; FF, fat fraction. 
Table III. Analysis of the effects of contrast agent.

\begin{tabular}{lcccc}
\hline Parameter & Organ & Estimate & CI.1 & CI.u \\
\hline $\mathrm{HU}_{\text {mixed }}$ & Spleen & 6.72 & 5.88 & 7.57 \\
& Liver & 3.80 & 2.17 & 5.42 \\
& Kidneys & 6.64 & 5.83 & 7.46 \\
& Fat tissue & -1.12 & -2.38 & 0.13 \\
$\mathrm{FF}$ & Muscle & -0.86 & -1.99 & 0.28 \\
& Spleen & -4.19 & -5.01 & -3.37 \\
& Liver & -1.94 & -3.21 & -0.68 \\
& Kidneys & -0.35 & -1.28 & 0.57 \\
& Fat tissue & 1.28 & 0.24 & 2.31 \\
& Muscle & 1.93 & 1.02 & 2.83 \\
$\mathrm{Q}_{\mathrm{e}}$ & Spleen & 0.0067 & 0.0059 & 0.0076 \\
& Liver & 0.0038 & 0.0022 & 0.0054 \\
& Kidneys & 0.0066 & 0.0058 & 0.0075 \\
& Fat tissue & -0.0011 & -0.0024 & 0.0001 \\
& Muscle & -0.0009 & -0.0020 & 0.0003 \\
$\mathrm{Z}_{\text {eff }}$ & Spleen & 1.07 & 1.03 & 1.11 \\
& Liver & 1.08 & 1.04 & 1.12 \\
& Kidneys & 2.51 & 2.45 & 2.57 \\
& Fat tissue & 0.45 & 0.38 & 0.52 \\
& Muscle & 0.20 & 0.15 & 0.25 \\
\hline
\end{tabular}

In each organ, the use of an iodine containing contrast agent was associated with a change in the variable values. The estimated change in the different parameters for each organ with the $95 \%$ confidence interval are given. CI.1, Lower confidence interval; CI.u, upper confidence interval; $\mathrm{HU}_{\text {mixed }}$, Hounsfield units derived from mixed tube voltages of $100 \mathrm{kVp}$ and $140 \mathrm{kVp}$ representing $120 \mathrm{kV}$; $\varrho_{e}$, electron density relative to water; $Z_{\text {eff }}$, effective atomic number, $F F$, fat fraction

parameter and organ, as well as the $95 \%$ confidence intervals. Taking every variable into account, muscle and fat tissue showed the lowest differences, whereas kidney and spleen showed the highest differences when non-contrast and contrast-enhanced results were compared.

Between-organ differences in the parameters were quantified separately for DECT scans performed with and without contrast agent. Results of the analyses of scans without contrast agent are shown in Table IV, whereas those with contrast agent are shown in Table V. Again, we report the estimated difference as well as the 95\% confidence intervals. Comparisons of the different organs within noncontrast DECT acquisitions revealed only low differences in $\mathrm{HU}_{\text {mixed }}$ between liver and muscle, and in $\mathrm{Z}_{\text {eff }}$ between liver, muscle, and spleen. On contrast-enhanced DECT scans, low differences were shown in $\mathrm{HU}_{\text {mixed }}$ between liver and spleen, in $\mathrm{Z}_{\text {eff }}$ between liver and spleen, and in FF between liver, spleen, and muscle. The value ranges given above showed large overlaps for the different tissues within the same acquisition. Overlaps are visualized in graphs in Figure 3. We also inserted the measured values of $\mathrm{HU}_{\text {mixed }}, \mathrm{Z}_{\text {eff }}$ and FF for liver, spleen, kidney and muscle in a 3D graph (Figure 2). Especially the overlaps of liver, spleen and muscle can be regarded here. In Figure 3 and Figure 4, fat
Table IV. Analysis of the differences in dual energy CT (DECT) parameters between organs without the use of contrast agent.

\begin{tabular}{|c|c|c|c|c|}
\hline Parameter & $\begin{array}{l}\text { Organs } \\
\text { compared }\end{array}$ & Estimate & CI.1 & CI.u \\
\hline \multirow[t]{10}{*}{$\mathrm{HU}_{\text {mixed }}$} & Liver - spleen & 4.45 & 3.37 & 5.53 \\
\hline & Kidneys - spleen & -22.18 & -22.83 & -21.53 \\
\hline & Fat tissue - spleen & -124.85 & -125.75 & -123.95 \\
\hline & Muscle - spleen & 3.39 & 2.51 & 4.27 \\
\hline & Kidneys - liver & -26.63 & -27.70 & -25.56 \\
\hline & Fat tissue - liver & -129.30 & -130.54 & -128.05 \\
\hline & Muscle - liver & -1.06 & -2.29 & 0.17 \\
\hline & Fat tissue - kidneys & -102.67 & -103.56 & -101.78 \\
\hline & Muscle - kidneys & 25.57 & 24.70 & 26.44 \\
\hline & Muscle - fat tissue & 128.24 & 127.16 & 129.31 \\
\hline \multirow[t]{10}{*}{$\mathrm{FF}$} & Liver - spleen & -2.76 & -3.64 & -1.88 \\
\hline & Kidneys - spleen & 12.48 & 11.71 & 13.25 \\
\hline & Fat tissue - spleen & 97.40 & 96.63 & 98.17 \\
\hline & Muscle - spleen & -4.95 & -5.72 & -4.18 \\
\hline & Kidneys - liver & 15.24 & 14.27 & 16.21 \\
\hline & Fat tissue - liver & 100.16 & 99.18 & 101.14 \\
\hline & Muscle - liver & -2.19 & -3.17 & -1.21 \\
\hline & Fat tissue - kidneys & 84.92 & 84.04 & 85.80 \\
\hline & Muscle - kidneys & -17.43 & -18.31 & -16.56 \\
\hline & Muscle - fat tissue & -102.35 & -103.24 & -101.47 \\
\hline \multirow{10}{*}{$\varrho_{\mathrm{e}}$} & Liver - spleen & 0.0044 & 0.0034 & 0.0055 \\
\hline & Kidneys - spleen & -0.0222 & -0.0228 & -0.0215 \\
\hline & Fat tissue - spleen & -0.1249 & -0.1258 & -0.1239 \\
\hline & Muscle - spleen & 0.0034 & 0.0025 & 0.0043 \\
\hline & Kidneys - liver & -0.0266 & -0.0277 & -0.0256 \\
\hline & Fat tissue - liver & -0.1293 & -0.1305 & -0.1281 \\
\hline & Muscle - liver & -0.0011 & 0.0023 & 0.0002 \\
\hline & Fat tissue - kidneys & -0.1027 & -0.1036 & -0.1018 \\
\hline & Muscle - kidneys & 0.0256 & 0.0247 & 0.0264 \\
\hline & Muscle - fat tissue & 0.1282 & 0.1272 & 0.1293 \\
\hline \multirow[t]{10}{*}{ Zeff } & Liver - spleen & -0.04 & -0.06 & -0.02 \\
\hline & Kidneys - spleen & 0.09 & 0.07 & 0.12 \\
\hline & Fat tissue - spleen & -1.83 & -1.87 & -1.79 \\
\hline & Muscle - spleen & -0.08 & -0.11 & -0.05 \\
\hline & Kidneys - liver & 0.13 & 0.11 & 0.16 \\
\hline & Fat tissue - liver & -1.79 & -1.83 & -1.75 \\
\hline & Muscle - liver & -0.04 & -0.07 & -0.01 \\
\hline & Fat tissue - kidneys & -1.92 & -1.97 & -1.88 \\
\hline & Muscle - kidneys & -0.17 & -0.21 & -0.14 \\
\hline & Muscle - fat tissue & 1.75 & 1.70 & 1.79 \\
\hline
\end{tabular}

The estimated change between organs and its $95 \%$ confidence interval are given for each parameter. CI.1, Lower confidence interval; CI.u, upper confidence interval; $\mathrm{HU}_{\text {mixed }}$, Hounsfield units derived from mixed tube voltages of $100 \mathrm{kVp}$ and $140 \mathrm{kVp}$ representing $120 \mathrm{kV} ; \varrho_{\mathrm{e}}$, electron density relative to water; $Z_{\text {eff }}$, effective atomic number; $F F$, fat fraction.

tissue was not included in the visualization since all values of fat were highly significant different from all other tissues.

Comparing the mean values in non-contrast DECT scans of $\mathrm{HU}_{\text {mixed }}$ only showed no significant differences by comparing spleen to muscle $(p=0.99)$. Mean values of $\varrho_{e}$ showed no significant differences by comparing liver to 
muscle ( $p=0.24), \mathrm{Z}_{\mathrm{eff}}$ by liver to spleen $(p=0.32)$ and liver to muscle $(p=0.16)$. For FF and all other tissue comparisons results showed statistically relevant differences $(p<0.05)$.

Comparison of the mean $\mathrm{HU}_{\text {mixed }}$ values in contrastenhanced scans showed no significant differences between liver to spleen $(p=0.96)$, whereas all other comparisons were highly significant $(p<0.001)$.

\section{Discussion}

This retrospective analysis of clinical dual-energy CT scans (DECT) reports a statistical summary of CT values for different abdominal tissues with and without the use of contrast agent in a 12-month cohort. Furthermore, we evaluated the estimated differences between abdominal organs, as well the effects of a contrast agent on different CT parameters. A current focus of interest in radiology is the idea of automated segmentation techniques that can automatically recognize different organs and tissues. Therefore, it is of great importance to gather data from many clinical study populations and determine the generally observed distributions to derive normal values. Furthermore, we were able to document the effects of contrast agent on the DECT values of abdominal organs. We are convinced that angiopathic-ischemic lesions with reduced blood circulation, or blood clots in the vascular system of an organ, lead to low uptake of contrast agent by the respective organ. Thus, estimating the effects of contrast agent on different DECT variables is of major interest.

By applying the DECT method with low- and high-energy spectra, we obtained additional parameters for tissue characterization, such as the $\mathrm{CT}$ number for mixed images at different tube voltages $\left(\mathrm{HU}_{\text {mixed }}\right)$, the electron density relative to water $\left(Q_{e}\right)$, the effective atomic number $\left(Z_{\text {eff }}\right)$, and the fat fraction (FF). In contrast, only $\mathrm{HU}$ values are generated on single-energy $\mathrm{CT}$, which is a disadvantage, because tissues are generally likely to show more differences if more variables are involved. Thus, we examined DECT scans to obtain more knowledge on organic structures and improve organ characterization.

We could determine the effects of the contrast agent on each DECT parameter in each organ. Whereas muscle and fat tissue showed lowest changes in contrast-enhanced scans for each parameter due to their low intake of contrast agent, the kidneys and spleen showed the highest changes of each included organ. This is most likely a result of both organs having a high blood circulation, but also because the CT protocol used was dedicated for work-up of the urinary track, and contrast agent was therefore administered using a split bolus technique. Consequently, our results support the assumption that pathologically ischemic organic changes are detectable using our DECT protocol. Depending on the units of the measured variables, $\mathrm{Z}_{\text {eff }}$ and $\mathrm{HU}_{\text {mixed }}$ were the most affected by contrast
Table V. Analysis of the differences in dual energy CT (DECT) parameters between organs with the use of contrast agent.

\begin{tabular}{|c|c|c|c|c|}
\hline Parameter & $\begin{array}{l}\text { Organs } \\
\text { compared }\end{array}$ & Estimate & CI.1 & CI.u \\
\hline \multirow[t]{10}{*}{$\mathrm{HU}_{\text {mixed }}$} & Liver - spleen & 1.50 & 0.02 & 2.98 \\
\hline & Kidneys - spleen & -22.29 & -23.27 & -21.31 \\
\hline & Fat tissue - spleen & -132.72 & -133.94 & -131.51 \\
\hline & Muscle - spleen & -4.22 & -5.32 & -3.11 \\
\hline & Kidneys - liver & -23.79 & -25.26 & -22.32 \\
\hline & Fat tissue - liver & -134.22 & -135.86 & -132.59 \\
\hline & Muscle - liver & -5.72 & -7.27 & -4.16 \\
\hline & Fat tissue - kidneys & -110.43 & -111.64 & -109.32 \\
\hline & Muscle - kidneys & 18.07 & 16.98 & 19.17 \\
\hline & Muscle - fat tissue & 128.51 & 127.20 & 129.81 \\
\hline \multirow[t]{10}{*}{ FF } & Liver - spleen & -0.52 & -1.75 & 0.70 \\
\hline & Kidneys - spleen & 16.32 & 15.36 & 17.29 \\
\hline & Fat tissue - spleen & 102.88 & 101.82 & 103.95 \\
\hline & Muscle - spleen & 1.19 & 0.24 & 2.13 \\
\hline & Kidneys - liver & 16.85 & 15.62 & 18.08 \\
\hline & Fat tissue - liver & 103.41 & 102.10 & 104.72 \\
\hline & Muscle - liver & 1.71 & 0.50 & 2.92 \\
\hline & Fat tissue - kidneys & 86.56 & 85.49 & 87.63 \\
\hline & Muscle - kidneys & -15.14 & -16.09 & -14.19 \\
\hline & Muscle - fat tissue & -101.70 & -102.75 & -100.64 \\
\hline \multirow[t]{10}{*}{ @e } & Liver - spleen & 0.0015 & 0.0000 & 0.0030 \\
\hline & Kidneys - spleen & -0.0223 & -0.0233 & -0.213 \\
\hline & Fat tissue - spleen & -0.1327 & -0.1339 & -0.1315 \\
\hline & Muscle - spleen & -0.0042 & -0.0053 & -0.0031 \\
\hline & Kidneys - liver & -0.0238 & -0.0253 & -0.0223 \\
\hline & Fat tissue - liver & -0.1342 & -0.1359 & -0.1326 \\
\hline & Muscle - liver & -0.0057 & -0.0073 & -0.0042 \\
\hline & Fat tissue - kidneys & -0.1104 & -0.1116 & -0.1092 \\
\hline & Muscle - kidneys & 0.0181 & 0.170 & 0.192 \\
\hline & Muscle - fat tissue & 0.1285 & 0.1272 & 0.1298 \\
\hline \multirow[t]{10}{*}{ Zeff } & Liver - spleen & -0.03 & -0.08 & 0.02 \\
\hline & Kidneys - spleen & 1.54 & 1.47 & 1.60 \\
\hline & Fat tissue - spleen & -2.45 & -2.52 & -2.37 \\
\hline & Muscle - spleen & -0.95 & -1.00 & -0.89 \\
\hline & Kidneys - liver & 1.56 & 1.50 & 1.63 \\
\hline & Fat tissue - liver & -2.42 & -2.49 & -2.34 \\
\hline & Muscle - liver & -0.92 & -0.97 & -0.86 \\
\hline & Fat tissue - kidneys & -3.98 & -4.06 & -3.90 \\
\hline & Muscle - kidneys & -2.48 & -2.55 & -2.41 \\
\hline & Muscle - fat tissue & 1.50 & 1.42 & 1.58 \\
\hline
\end{tabular}

The estimated change between organs and its $95 \%$ confidence interval are given for each parameter. CI.1, lower confidence interval; CI.u, upper confidence interval; $\mathrm{HU}_{\text {mixed }}$, Hounsfield units derived from mixed tube voltages of $100 \mathrm{kVp}$ and $140 \mathrm{kVp}$ representing $120 \mathrm{kV} ; \varrho_{\mathrm{e}}$, electron density relative to water; $\mathrm{Z}_{\text {eff }}$, effective atomic number; $\mathrm{FF}$, fat fraction.

agent because both parameters are affected by the atomic number of elements. Thus, the effects of the iodine-based (atomic number of iodine, $\mathrm{Z}=53$ ) contrast agent were measurable in the collected DECT variables. We generated the 95\% confidence intervals for each organ and DECT variable before and after contrast agent application, which allows the 

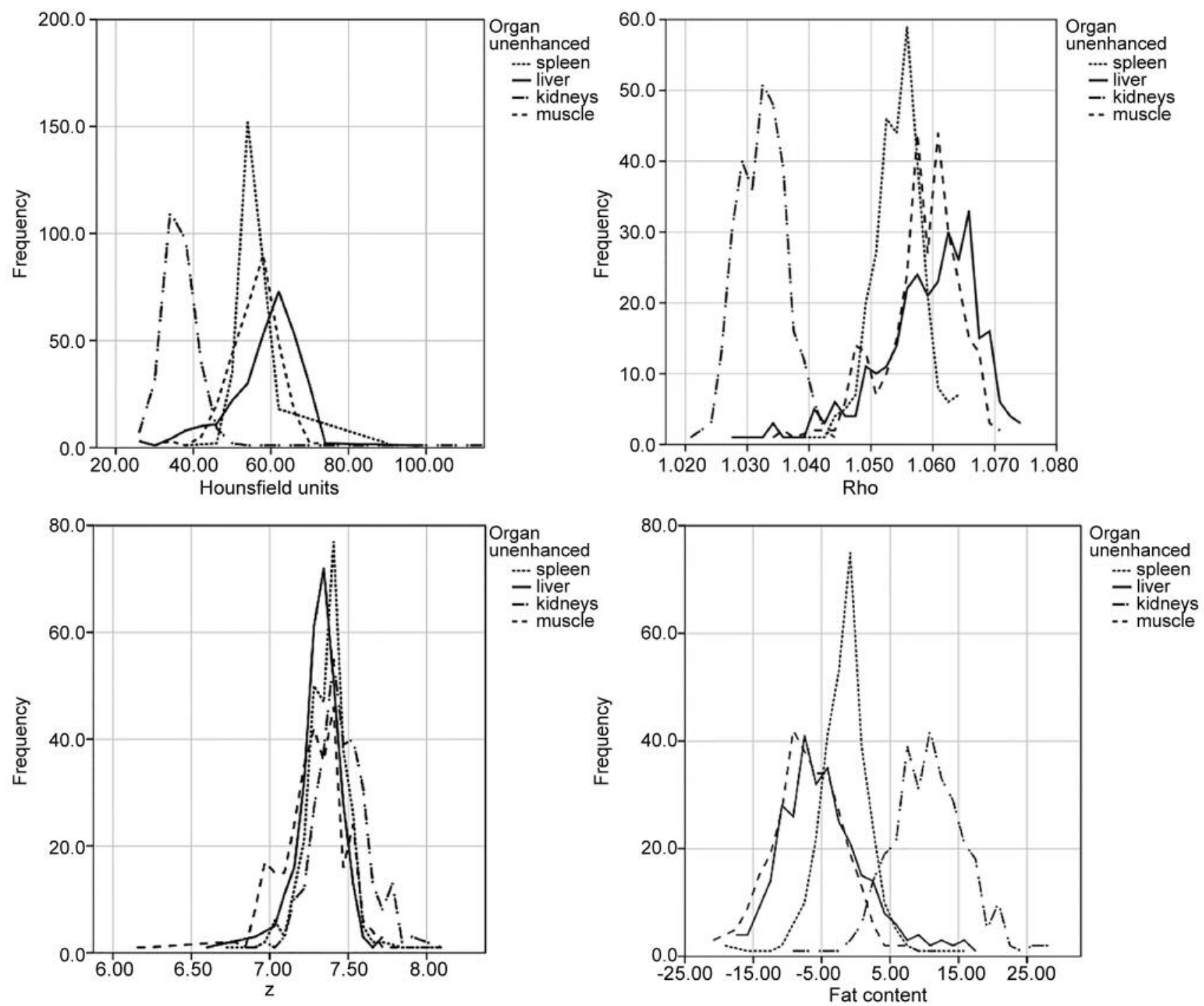

Figure 3. Visualized overlaps of measured values for the different tissues in non-contrast DECT scans. Graphs showing the measurements of the parameters $H U_{\text {mixed }}(C T$ values of mixed images in $H U$ units), Rho (electron density relative to water), $Z$ (effective atomic number) and fat fraction (in percent of the soft tissue). Since fat tissue shows different value ranges for all parameters it is not demonstrated here.

values obtained from patients to be checked, and those patients with values lower or higher than the confidence interval should be observed more closely if pathological changes in the vascular system feeding or within the organ exist. We also measured an effect of contrast agent on the FF. Although this parameter is derived from the material decomposition algorithm of the syngo.via ${ }^{\circledR}$ application profile "Liver VNC", which extracts the iodine and creates virtual non-contrast images, we still noticed differences in the FF in response to contrast agent administration. We assume that these differences are an error due to contrast agent application, with the software not fully extracting the iodine attenuation from the virtual noncontrast images.
Our study also revealed that the variable $\varrho_{e}$ is not an ideal parameter to work with alone for tissue characterisation. Application of the formula described in Saito et al. (21) results in very small parameter values. Thus, differences can to some extent only be recognized to the third decimal place. Furthermore, because $\varrho_{e}$ is directly derieved from HU, it contributes no further information about the organic structure of tissue.

Former studies have already shown that DECT facilitates the differentiation of different materials $(7,8,10,15-17,22$, 23). However, these earlier studies referred predominantly to the differentiation of materials with different compositions, for example kidney stones from uric acid or calcium $(17,24$, 


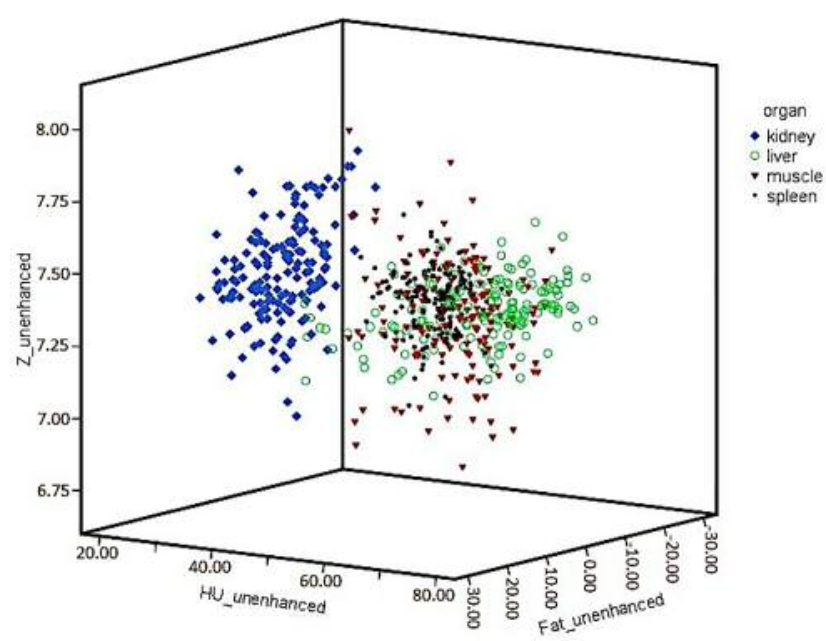

Figure 4. Visualization in a $3 D$ graph of the measured values of the parameters. HU, Hounsfield unit mixed from tube voltages $100 \mathrm{kVp}$ and $140 \mathrm{kVp} ; \mathrm{Z}$, effective atomic number; Fat, fat fraction of the tissues kidney, liver, muscle and spleen for non-contrast DECT scans.

25). The differentiation of soft tissues is an ambitious idea and may not yet be possible $(10,22)$ because soft tissues like liver, kidney, spleen, muscle, and fat tissue consist primarily of hydrogen, carbon, nitrogen and oxygen, which all have similar low average atomic numbers and consequently show no major differences in attenuation of X-rays of different energy levels $(16,22)$. However, even when materials show similar attenuation, they can still show different electron densities and elemental compositions, which may lead to their separation based on material qualities $(7,8)$. Therefore, we tested whether soft tissues can be separated from each other using the aforementioned DECT parameters or the averaged Hounsfield units obtained from mixing of the two different energy spectra. By measuring values in a patient cohort collected over the course of a year, we were able to estimate differences and confidence intervals, within which the true differences between organs lie. After comparing all collected variable values for the spleen, liver, and muscle, we found only low differences on non-contrast images. Nevertheless, the contrast-enhanced DECT scans showed greater differences, with the variable values extracted from muscle showing great differences from the values of liver and spleen because of the low contrast uptake of muscle. Thus, automated organ detection would be possible by applying contrast agent. Nevertheless, when comparing the liver to spleen, we only detected small differences in FF and $\mathrm{Z}_{\text {eff }}$ in non-contrast images, and also in $\mathrm{HU}_{\text {mixed }}$, $\mathrm{FF}$, and $\mathrm{Z}_{\text {eff }}$ in contrast-enhanced images. The estimated differences in $\mathrm{HU}_{\text {mixed }}$ between liver and spleen were higher in noncontrast images than in contrast-enhanced images. Nevertheless, we are convinced that there is potential to increase the differences between these tissues in contrastenhanced images, by developing the CT protocol and the contrast agent application. For example, the spleen and liver should show greater differences in images acquired during arterial phase $\mathrm{CT}$.

A recent study by Hunemohr et al. also measured $\mathrm{HU}, \varrho_{\mathrm{e}}$, and $\mathrm{Z}_{\text {eff }}$ on DECT scans (16). They performed scans at 120 $\mathrm{kV}$ and measured the parameters three times in kidney, liver, muscle, and three different fat tissues. The $\mathrm{HU}$ values ranged from 43 to $63 \mathrm{HU}$ for liver, 41 to $46 \mathrm{HU}$ for kidney, 40 to 44 $\mathrm{HU}$ for muscle, and -98 to $-55 \mathrm{HU}$ for fat tissue. The values for $\varrho_{\mathrm{e}}$ were 1.05-1.07 for liver, 1.05 for kidney, 1.05 for muscle, and $0.93-0.97$ for fat tissue. $Z_{\text {eff }}$ ranged from 7.26 to 7.30 for liver, 7.27 to 7.36 for kidney, 7.21 to 7.31 for muscle, and 6.10 to 6.48 for fat tissue. Their values for $\varrho_{e}$ and $\mathrm{Z}_{\text {eff }}$ are similar to those in our study because the tube voltage of $120 \mathrm{kV}$ corresponds with our mixed $100 \mathrm{kV}$ and $140 \mathrm{Sn}$ $\mathrm{kV}$ images. Nevertheless, the study sample of Hunemohr et $a l$. was smaller than ours and did not aim to generate normal distributions. By taking measurements from a large sample, as in our study, it can be assumed that patients with values around the median are likely to correspond to healthy adults. Additionally, we calculated the first and third quartiles, as pathological organic changes could possibly be present in patients with values below or above these limits.

Our study has several limitations. First even if we fixed tube voltages, the scanner was allowed for tube current modulation since we used clinical protocols for our retrospective analyses. The aim of the algorithm is to maintain a global image noise level, but we could not take into consideration dose variations and its effect on the different parameters evaluated. Second our standardized scan protocol included a split bolus administration of contrast medium, e.g., two separate following contrast peaks that might have influenced organ attenuation due to overlap of early and late contrast phases. Third our normal distribution dataset was generated using vendor specific software tools that may be difficult to transfer to other technical equipment.

To our knowledge our work is the first attempt to generate normal values for parenchymatous abdominal organs using a standardized dual energy CT imaging protocol. Our data was aimed at multiparametric evaluation that enables multidimensional analysis. Such data may be crucial for the development of deep learning powered algorithms that allow for automatic tissue characterization. In future studies, we aim to examine whether measurements of the variables $\mathrm{HU}_{\text {mixed }}, \mathrm{Z}_{\text {eff }}$, and $\mathrm{FF}$ are suitable for diagnosing disease. Studies involving the collaboration of different institutes of pathology should be pursued. As a histological examination is the gold standard for diagnosis in many diseases, we should perform correlation analyses between the histological results and radiological examinations. It is conceivable that the microscopic changes a disease can cause may also affect 
the measured parameters. Diseases leading to increased organ density, such as fibrosis or cirrhosis of the liver, should consequently lead to higher CT numbers and $\varrho_{e}$, whereas diseases with lower organ density such as liver steatosis, should lead to lower CT numbers and $\varrho_{e}$, but a higher FF.

\section{Conclusion}

Organ characterization using multiparametric dual energy CT analysis is possible. The measurable CT parameters showed differences before and after contrast agent application and may, therefore, be useful for detecting pathological vascular changes. The increased number of parameters obtained from DECT in comparison to single-energy CT led to improved organ characterization and shows promise for future automated segmentation techniques. To our knowledge this is the first attempt to provide normal values for characterization of parenchymatous organs.

\section{Conflicts of Interest}

The Authors declare no conflicts of interest.

\section{Authors' Contributions}

Diana Kreul (first author): Study design, literature research, data analysis, editing and writing of the article. Tilo Niemann (corresponding author): Literature research, editing, writing and proofreading of the article. Rahel Kubik: Data analysis and proofreading of the article. John Froehlich and Michael Thali: Coediting, proofreading of the article. All Authors made pertinent contributions to the article, and proofread and approved the final article before submission.

\section{References}

1 Hounsfield GN: Computerized transverse axial scanning (tomography). 1. Description of system. Br J Radiol 46(552): 1016-1022, 1973. PMID: 4757352. DOI: 10.1259/0007-128546-552-1016

2 Ambrose J: Computerized transverse axial scanning (tomography). 2. Clinical application. Br J Radiol 46(552): 1023-1047, 1973. PMID: 4757353. DOI: 10.1259/0007-128546-552-1023

3 Rutherford RA, Pullan BR and Isherwood I: X-ray energies for effective atomic number determination. Neuroradiology 11(1): 23-28, 1976. PMID: 934469. DOI: 10.1007/BF00327254

4 Rutherford RA, Pullan BR and Isherwood I: Measurement of effective atomic number and electron density using an EMI scanner. Neuroradiology 11(1): 15-21, 1976. PMID: 934468. DOI: $10.1007 / \mathrm{BF} 00327253$

5 Kruger RA, Riederer SJ and Mistretta CA: Relative properties of tomography, K-edge imaging, and K-edge tomography. Med Phys 4(3): 244-249, 1977. PMID: 329091. DOI: 10.1118/1.594374

6 Riederer SJ and Mistretta CA: Selective iodine imaging using K-edge energies in computerized x-ray tomography. Med Phys 4(6): 474-481, 1977. PMID: 927384. DOI: 10.1118/1.594357
7 Landry G, Reniers B, Granton PV, van Rooijen B, Beaulieu L, Wildberger JE and Verhaegen F: Extracting atomic numbers and electron densities from a dual source dual energy CT scanner: experiments and a simulation model. Radiother Oncol 100(3): 375-379, 2011. PMID: 21924780. DOI: 10.1016/j.radonc. 2011.08.029

8 Landry G, Seco J, Gaudreault M and Verhaegen F: Deriving effective atomic numbers from DECT based on a parameterization of the ratio of high and low linear attenuation coefficients. Phys Med Biol 58(19): 6851-6866, 2013. PMID: 24025623. DOI: 10.1088/0031-9155/58/19/6851

9 Chiro GD, Brooks RA, Kessler RM, Johnston GS, Jones AE, Herdt JR and Sheridan WT: Tissue signatures with dual-energy computed tomography. Radiology 131(2): 521-523, 1979. PMID: 441344. DOI: 10.1148/131.2.521

10 Johnson TR, Krauss B, Sedlmair M, Grasruck M, Bruder H, Morhard D, Fink C, Weckbach S, Lenhard M, Schmidt B, Flohr T, Reiser MF and Becker CR: Material differentiation by dual energy CT: initial experience. Eur Radiol 17(6): 1510-1517, 2007. PMID: 17151859 . DOI: $10.1007 / \mathrm{s} 00330-006-0517-6$

11 Petersilka M, Bruder H, Krauss B, Stierstorfer K and Flohr TG: Technical principles of dual source CT. Eur J Radiol 68(3): 362368, 2008. PMID: 18842371. DOI: 10.1016/j.ejrad.2008.08.013

12 Millner MR, McDavid WD, Waggener RG, Dennis MJ, Payne WH and Sank VJ: Extraction of information from CT scans at different energies. Med Phys 6(1): 70-71, 1979. PMID: 440238. DOI: $10.1118 / 1.594555$

13 Marin D, Boll DT, Mileto A and Nelson RC: State of the art: dual-energy CT of the abdomen. Radiology 271(2): 327-342, 2014. PMID: 24761954. DOI: 10.1148/radiol.14131480

14 Alvarez RE and Macovski A: Energy-selective reconstructions in X-ray computerized tomography. Phys Med Biol 21(5): 733744, 1976. PMID: 967922. DOI: 10.1088/0031-9155/21/5/002

15 Fornaro J, Leschka S, Hibbeln D, Butler A, Anderson N, Pache G, Scheffel H, Wildermuth S, Alkadhi H and Stolzmann P: Dualand multi-energy CT: approach to functional imaging. Insights Imaging 2(2): 149-159, 2011. PMID: 22347944. DOI: 10.1007/ s13244-010-0057-0

16 Hünemohr N, Paganetti H, Greilich S, Jäkel O and Seco J: Tissue decomposition from dual energy CT data for MC based dose calculation in particle therapy. Med Phys 41(6): 061714, 2014. PMID: 24877809. DOI: 10.1118/1.4875976

17 Malusek A, Karlsson M, Magnusson M and Carlsson GA: The potential of dual-energy computed tomography for quantitative decomposition of soft tissues to water, protein and lipid in brachytherapy. Phys Med Biol 58(4): 771-785, 2013. PMID: 23322084. DOI: $10.1088 / 0031-9155 / 58 / 4 / 771$

18 Avrin DE, Macovski A and Zatz LE: Clinical application of Compton and photo-electric reconstruction in computed tomography: preliminary results. Invest Radiol 13(3): 217-222, 1978. PMID: 711396. DOI: 10.1097/00004424-197805000-00007

19 Mileto A, Marin D, Alfaro-Cordoba M, Ramirez-Giraldo JC, Eusemann CD, Scribano E, Blandino A, Mazziotti S and Ascenti G: Iodine quantification to distinguish clear cell from papillary renal cell carcinoma at dual-energy multidetector CT: a multireader diagnostic performance study. Radiology 273(3): 813-820, 2014. PMID: 25162309. DOI: 10.1148/radiol. 14140171

20 Martin SS, Weidinger S, Czwikla R, Kaltenbach B, Albrecht MH, Lenga L, Vogl TJ and Wichmann JL: Iodine and fat 
quantification for differentiation of adrenal gland adenomas from metastases using third-generation dual-source dual-energy computed tomography. Invest Radiol 53(3): 173-178, 2018. PMID: 28990974. DOI: 10.1097/RLI.0000000000000425

21 Saito M and Sagara S: A simple formulation for deriving effective atomic numbers via electron density calibration from dual-energy CT data in the human body. Med Phys 44(6): 22932303, 2017. PMID: 28236659. DOI: 10.1002/mp.12176

22 Agrawal MD, Pinho DF, Kulkarni NM, Hahn PF, Guimaraes AR and Sahani DV: Oncologic applications of dual-energy CT in the abdomen. Radiographics 34(3): 589-612, 2014. PMID: 24819783. DOI: $10.1148 / \mathrm{rg} .343135041$

23 Bazalova M, Carrier JF, Beaulieu L and Verhaegen F: Dualenergy CT-based material extraction for tissue segmentation in Monte Carlo dose calculations. Phys Med Biol 53(9): 2439-2456, 2008. PMID: 18421124. DOI: 10.1088/0031$9155 / 53 / 9 / 015$
24 Ananthakrishnan L, Duan X, Xi Y, Lewis MA, Pearle MS, Antonelli JA, Goerne H, Kolitz EM, Abbara S, Lenkinski RE, Fielding JR and Leyendecker JR: Dual-layer spectral detector CT: non-inferiority assessment compared to dual-source dualenergy $\mathrm{CT}$ in discriminating uric acid from non-uric acid renal stones ex vivo. Abdom Radiol (NY) 43(11): 3075-3081, 2018. PMID: 29626256. DOI: 10.1007/s00261-018-1589-x

25 Franken A, Gevenois PA, Muylem AV, Howarth N and Keyzer $\mathrm{C}$ : In vivo differentiation of uric acid versus non-uric acid urinary calculi with third-generation dual-source dual-energy CT at reduced radiation dose. AJR Am J Roentgenol 210(2): 358363, 2018. PMID: 29166148. DOI: 10.2214/AJR.17.18091

Received June 1, 2021

Revised June 19, 2021

Accepted July 27, 2021 